Pacific Journal of Mathematics

THE TRANSLATION GROUPS OF $\boldsymbol{n}$-UNIFORM TRANSLATION 


\title{
THE TRANSLATION GROUPS OF $n$-UNIFORM TRANSLATION HJELMSLEV PLANES
}

\author{
DAVID A. DRAKE
}

The purpose of this paper is twofold: first, to determine the full translation groups for all $n$-uniform translation affine Hjelmslev planes for all positive integers $n$; and second, to prove that all such groups occur as the full translation groups of Pappian Hjelmslev Planes.

1. Introduction. For brevity's sake, we introduce the following three conventions: Hjelmslev plane will be abbreviated to $H$-plane; we will always mean affine (rather than projective) when we write translation $H$-plane; and throughout the paper, translation group will denote the group of all translations. H. Lüneburg has previously defined translation $H$-planes [7] and has determined the translation groups of all uniform translation $H$-planes [7, Satz 8.3]. The author has defined a class of finite $H$-planes called $n$-uniform $H$-planes in such a way that the finite uniform $H$-planes are just the $n$-uniform $H$-planes with $n=1$ and 2 [3]. In $\S 2$, we prove (see Theorem 2.6.) that only certain groups can occur as translation groups of $n$-uniform translation $H$-planes; and in $\S 3$, we establish the converse. As algebraic corollaries to the geometric theorem of $\S 2$, we obtain results on the additive structures of the finite Desarguesian $H$-rings. (See Corollary 3.1 and Remark 3.3.). This is possible, because every Desarguesian $H$-ring coordinatizes a Desarguesian affine $H$-plane, because every Desarguesian affine $H$-plane is a translation $H$-plane, and because every finite Desarguesian $H$-plane is $n$-uniform for some $n$.

In $\S 3$, we quote a result of W. E. Clark and the author on the additive structure of finite commutative Desarguesian $H$-rings; we use this result to show that all groups permitted by Theorem 2.6 do in fact occur as translation groups of Pappian affine $H$-planes. Then every translation group of an $n$-uniform translation $H$-plane $A$ is isomorphic to the translation group of a Pappian affine $H$-plane $B$. One may always take $B$ to have the same invariants as $A$. One may also always choose $B$ so that its associated ordinary affine plane has prime order.

2. The translation groups of finite translation $H$-planes. The reader is referred to $P$. Dembowski [2] or to the papers in the bibliography for definitions of affine and projective $H$-planes. We will write $P \sim Q, g \nsim h$, etc., to mean the point $P$ is neighbor to $Q$, the line $g$ is not neighbor to $h$, etc. Associated with every finite 
affine or projective $H$-plane are two invariants denoted by $s$ and $t$. We may take $t$ to be the number of lines through a point $P$ which are neighbor to the line $g$ where $(P, g)$ is an arbitrary flag of the $H$-plane; then $s+t$ will denote the total number of lines incident with $P$. It is well known that $s / t$ is the order of the ordinary affine or projective plane associated with the $H$-plane. (See [4] and [7].)

Definition 2.1. Let $P$ be a point of an $H$-plane $\pi$. We define $\bar{P}$ to be the following incidence structure. The points of $\bar{P}$ are the points $Q$ of $\pi$ such that $Q \sim P$. The lines of $\bar{P}$ are the nonempty point sets $l^{P}=l \cap \bar{P}, l$ being a line of $\pi$. Incidence is given by inclusion.

DeFinition 2.2. We define a 1-uniform affine (projective) $H$-plane to be a finite ordinary affine (projective) plane. We call a finite affine or projective $H$-plane $n$-uniform $(n \geqq 2)$ provided that

(a) $\bar{P}$ is an $(n-1)$-uniform affine $H$-plane for each point $P$ in $\pi$.

(b) For each $\bar{P}$, every line $l^{P}$ is the restriction of the same number of lines from $\pi$.

The following result is part of [3, Proposition 2.2]. The reader should thoroughly acquaint himself with the content of this proposition as it will be used frequently in the rest of the paper.

Proposition 2.1. Let $\pi$ be an n-uniform projective or affine $H$-plane. Then $\pi$ satisfies the following properties:

(1) If $r=s / t$, then $s=r^{n}$ and $t=r^{n-1}$.

(2) Distinct intersecting neighbor lines of $\pi$ meet in $r^{i}$ points for some integer $i$ such that $1 \leqq i \leqq n-1$.

(3) The dual of (2) holds in $\pi$.

(4) If $P \in h$, the number of lines through $P$ which intersect $h$ in $r^{i}$ or more points is $r^{n-i}$ for $i=1,2, \cdots, n$.

(5) The dual of (4) holds in $\pi$.

We write " $P(\cong i) Q$ " and read " $P$ is $i$-equivalent to $Q$ " to mean $P$ is joined to $Q$ by exactly $r^{i}$ lines; we write " $P(\sim i) Q$ " and read " $P$ is at least $i$-equivalent to $Q$ " to mean $P$ is joined to $Q$ by $\mathrm{r}^{i}$ or more lines.

(6) ( $\sim i)$ is an equivalence relation on points for $i=0,1, \cdots, n$.

(7) The following conditions imply $|l \cap k|>1: R, Q \in l ; R, S \in$ $k ; R(\cong i) Q ; Q(\sim i+1) S ; i$ is a nonnegative integer $<n$.

(8) If $P$ is any point of $\pi$, the number of points $Q$ of $\pi$ such that $Q(\sim i) P$ is $r^{2(n-i)}$ for $i=1,2, \cdots, n$.

In light of Proposition 2.1 (1), an $n$-uniform $H$-plane may be 
thought of as having three invariants $r, s$, and $t$. However, $s$ and $t$ are determined by $r$ and $n$; and thus, we shall write the invariant of an $n$-uniform $H$-plane $\pi$ to refer to $r$. Since $r=s / t$, the invariant of $\pi$ is the order of the ordinary affine or projective plane associated with $\pi$. Next we prove

Lemma 2.2. Let $P, Q, R$ be points of an n-uniform $H$-plane which satisfy $P, Q \in g ; P, R \in h ; Q, R \in k$. Further suppose $P(\cong i) Q(\cong i) R(\cong i) P$, $i<n$, and $g \nsim h$. Then $h \nsim k \nsim g$.

Proof. Proposition 2.1 (5) implies the number of points $X$ such that $X \in g$ and $X(\sim i+1) P$ is $r^{n-i-1}$. By (7), any line joining $R$ to such an $X$ is neighbor to $h$, hence not neighbor to $g$. Then no line joins $R$ to two such $X$. By (6), the number of lines joining $R$ to each such $X$ is $r^{i}$. Then the number of lines joining $R$ to all such $X$ is $r^{n-1}=t$. Thus all lines through $R$ which are neighbor to $h$ meet $g$ in points $X$ which satisfy $X(\sim i+1) P$. Then $k \nsim h$, and by symmetry $k \nsim g$.

To state the next several lemmas, we need some notation and a definition. We will write ${ }^{i} P$ to denote $\{Q: Q(\sim n-i) P\}$. Thus ${ }^{\circ} P=$ $\{P\}$ and ${ }^{n} P$ is the set of all points of the $H$-plane.

Definition 2.3. A mapping $\sigma$ defined on the point set of an affine $H$-plane is called a dilatation if the following condition is satisfied: $P, Q \in g ;(P) \sigma \in h ; g \| h$ imply $(Q) \sigma \in h$.

LEMma 2.3. Let $\sigma$ be a dilatation of an n-uniform affine $H$-plane. Let $P, Q, R, T$ be points such that $Q(\cong j) P(\cong j) R, T(\sim j+1) P$, and $(P) \sigma(\cong i+j)(Q) \sigma$. Then
(a) $(R) \sigma(\cong i+j)(P) \sigma$, and
(b) $\quad(T) \sigma(\sim i+j+1)(P) \sigma$ if $i+j<n$.

Proof. Let $g$ be any line through $P$ and $Q, h$ be any line through $P$ which is not neighbor to $g$. We first prove the lemma for all $R, T \in h$ such that $R(\cong j) P, T(\sim j+1) P$. We have $R, T(\sim j) Q$. Since $h \nsim g$, Proposition 2.1 (7) implies $R(\cong j) Q$. Let $k$ be any line through $R$ ane $Q, m$ be any line through $T$ and $Q$. By Lemma 2.2, $|h \cap k|=$ 1. Let $g^{\prime}, h^{\prime}$ be the lines through $(P) \sigma$ parallel respectively to $g, h$; let $k^{\prime}, m^{\prime}$ be the lines through $(Q) \sigma$ parallel respectively to $k, m$. In one form of the definition of affine $H$-planes (See [7] or [3], not [2].), the following condition is assumed: $|h \cap k|=1$ and $k \| k^{\prime}$ imply $\left|h \cap k^{\prime}\right|=1$. Then also $\left|h^{\prime} \cap k^{\prime}\right|=1$. Similarly, $\left|h^{\prime} \cap g^{\prime}\right|=1$; and since $|m \cap g|>1,\left|m^{\prime} \cap g^{\prime}\right|>1$. Since $(P) \sigma(\cong i+j)(Q) \sigma$ and $h^{\prime} \nsim k^{\prime}$, Pro- 
position 2.1 (7) implies $(R) \sigma(\sim i+j)(P) \sigma$. Since $m^{\prime} \sim g^{\prime} \nsim h^{\prime}$, the same argument implies $(T) \sigma(\sim i+j)(P) \sigma$. We have $(R) \sigma(\cong i+j)(P) \sigma$, for otherwise the above argument would yield $(Q) \sigma(\sim i+j+1)(P) \sigma$. Next, suppose $(T) \sigma(\cong i+j)(P) \sigma$. Then since $h^{\prime} \not g^{\prime},(T) \sigma(\cong i+j)(Q) \sigma$. If $i+j<n$, Lemma 2.2 implies $m^{\prime} \nsim g^{\prime}$. By the contradiction, we conclude that $(T) \sigma(\sim i+j+1)(P) \sigma$. To see that the conclusions of the lemma hold for points $R$ and $T$ on a line $h$ through $P$ such that $h \sim g$, we apply the above results, replacing $g$ and $Q$ by $g^{*}$ and $Q^{*}$ where $Q^{*} \in g^{*} \nsim g$ and $Q^{*}(\cong j) P$. (The existence of such a point $Q^{*} \in g^{*}$ is assured by Proposition 2.1 (5).)

LEMMA 2.4. Let $\sigma$ be a dilatation of an n-uniform affine $H$-plane. Let $(P) \sigma(\cong i)(Q) \sigma$ for nonneighbor points $P, Q$. Then if $k \leqq n-i$, $\left({ }^{n-k} P\right) \sigma={ }^{n-k-i}((P) \sigma)$.

Proof. Taking $j=0$ in Lemma 2.3 yields $\left({ }^{n} P\right) \sigma \subset{ }^{n-i}((P) \sigma)$ and $\left({ }^{n-1} P\right) \sigma \subset \subset^{n-i-1}((P) \sigma)$. It follows from Proposition 2.1 (8) that for each $k=0,1, \cdots, n-1$, there exists a point $R_{k}$ such that $R_{k}(\cong k) P$. Using induction and Lemma 2.3, we get $\left({ }^{n-k} P\right) \sigma \subset{ }^{n-i-k}((P) \sigma)$ for all $k \leqq n-i$. If we can prove that the last containment is equality when $k=0$, then another induction proof using Lemma 2.3 will yield the full conclusion of Lemma 2.4. Thus it suffices to prove that ${ }^{n-i}((P) \sigma) \subset$ Image $(\sigma)$.

We let $g$ denote the line joining $P$ and $Q ; g^{\prime}$, the line through $(P) \sigma$ which is parallel to $g$. Let $h^{\prime}$ be any line through $(P) \sigma$ not neighbor to $g^{\prime}$, and let $R^{\prime}$ be any point of $h^{\prime}$ satisfying $R^{\prime}(\sim i)(P) \sigma$. Let $k^{\prime}$ be any line joining $R^{\prime}$ to $(Q) \sigma$. Since $(Q) \sigma(\cong i)(P) \sigma$ and $R^{\prime}(\sim i)(P) \sigma, R^{\prime}(\sim i)(Q) \sigma$. Since $h^{\prime} \nsim g^{\prime}, R^{\prime}(\cong i)(Q) \sigma$. If $R^{\prime}(\sim i+1)(P) \sigma$, then $k^{\prime} \sim g^{\prime}$; hence $k^{\prime} \nsim h^{\prime}$. If $R^{\prime}(\cong i)(P) \sigma$, Lemma 2.2 implies $k^{\prime} \nsim h^{\prime}$. Then in all cases $\left|k^{\prime} \cap h^{\prime}\right|=1$. Let $h$ be the line through $P$ which is parallel to $h^{\prime}, k$ be the line through $Q$ which is parallel to $k^{\prime}$. Then $|k \cap h|=1$. If $\{R\}$ is $k \cap h$, then $(R) \sigma=R^{\prime}$. To see that Image $(\sigma)$ contains points $R^{\prime}$ on lines $h^{\prime} \sim g^{\prime}$, repeat the above argument using (in place of $g$ and $Q$ ) a line $g^{*}$ through $P$ such that $g^{*} \nsim g$ and a point $Q^{*}$ on $g^{*}$ such that $Q^{*} \nsim P$. Lemma 2.3 implies $(P) \sigma(\cong i)\left(Q^{*}\right) \sigma$. Since $g^{*} \nsim g$, a previous argument implies $\left(g^{*}\right)^{\prime} \nsim g^{\prime}$, hence $\left(g^{*}\right)^{\prime} \nsim h^{\prime}$. (Here $\left(g^{*}\right)^{\prime}$ denotes the line through $(P) \sigma$ which is parallel to $g^{*}$.) Then ${ }^{n-i}((P) \sigma) \subset$ Image $(\sigma)$, and the proof of the lemma is complete.

Lemmas 2.3 and 2.4 combine to yield

Proposition 2.5. Let $\sigma$ be a dilatation of an n-uniform affine $H$-plane. Let $P(\cong j) Q$ and $(P) \sigma(\cong i+j)(Q) \sigma$ for some $j<n-i$. 
Then for all $k \leqq n-i,\left({ }^{n-k} P\right) \sigma={ }^{n-k-i}((P) \sigma)$.

The reader is referred to [7] or [2] for the definition of translation $H$-planes and for the results on translation $H$-planes which we quote and use below. If $\pi$ is a set of subgroups (called components) of the group $T, \boldsymbol{J}(T, \pi)$ denotes the incidence structure with parallel relation defined as follows: the points are the elements of $T$; the lines are the right cosets of the components; incidence is given by inclusion; and lines are parallel if and only if they are cosets of the same component of $\pi$. If $A$ is any translation $H$-plane and if $T^{*}$ is the translation group of $A$, then $T^{*}$ is abelian and there exist $T, \pi$ such that $A \cong J(T, \pi)$ and $T^{*} \cong T$. Every element $t^{*}$ of $T^{*}$ may be defined on $T$ by $(x) t^{*}=x+t$ for all $x \in T$, some fixed $t \in T$. If $J(T, \pi)$ is an affine $H$-plane $A$ and if $T$ is abelian, then $A$ is a translation $H$-plane with translation group isomorphic to $T$. Finally, we note that the invariant of $A$ must be a prime power, since the ordinary affine plane associated with $A$ is a translation plane.

THEOREm 2.6. Let $A$ be an n-uniform translation $H$-plane with invariant $r=p^{x}$ and translation group $T^{*}$. Then there exist nonnegative integers $k_{1}, k_{2}, j$ such that $T^{*}$ is the direct sum of $2 x k_{1}$ cyclic subgroups of order $p^{j}$ and of $2 x k_{2}$ cyclic subgroups of order $p^{j+1}$.

Proof. We represent $A$ by $\boldsymbol{J}(T, \pi)$ where $T \cong T^{*}$. Let ${ }^{i} T$ denote the set of all elements of $T$ in ${ }^{i} 0$. Let $\tau \in{ }^{i} T$, $\tau^{*}$ denote the translation which adds $\tau$ to each element of $T$. Then all lines connecting 0 and $\tau$ are "traces" of $\tau^{*}$, i.e., are fixed by $\tau^{*}$. Then if $\beta \in T$, all lines through $\beta$ parallel to these traces are also traces of $\tau^{*}$, hence contain $(\beta) \tau^{*}$. Then $\beta(\sim n-i)(\beta) \tau^{*}$; and if $\beta \in{ }^{i} T, \tau+\beta=(\beta) \tau^{*} \in{ }^{i} T$. Then ${ }^{i} T$ is a subgroup of $T$. Let ${ }^{i} \pi$ denote the set of all intersections of ${ }^{i} T$ with components of $\pi$. Then ${ }^{i} 0$ is isomorphic to $J\left({ }^{i} T,{ }^{i} \pi\right)$. Since $A$ is $n$-uniform, ${ }^{i} 0$ is an $i$-uniform affine $H$-plane; since ${ }^{i} T$ is an abelian group, ${ }^{i} 0$ is a translation $H$-plane.

We prove the theorem by induction on $n$. The 1-uniform translation $H$-planes are just the finite translation planes, and it is well known that such planes have elementary abelian translation groups. Since the order of the translation group of such a plane equals $r^{2}$, the number of points in the plane, the theorem is satisfied with $j=1=k_{1}$ and $k_{2}=0$. Now let $A$ be an $n$-uniform translation $H$-plane with $n>1$. By the induction hypothesis, ${ }^{n-1} T$ is the direct sum of $2 x k_{1}$ cyclic subgroups of order $p^{j}$ and of $2 x k_{2}$ cyclic subgroups of order $p^{j+1}$ for suitable $k_{1}, k_{2}, j$. We may assume $j>0, k_{1}>0$. Let $\sigma$ be the dilatation of $A$ defined by $(\beta) \sigma=p \beta$ for all $\beta \in T$. By Lemma 2.4, Image $(\sigma)={ }^{i} 0$ for some $i<n$. If $i=0, T$ is elementary 
abelian. The theorem is then satisfied with $j=1, k_{1}=n, k_{2}=0$, since the number of points in $A$ is $r^{2 n}$.

Henceforth, we assume $i>0$. Since $i<n$, we may apply the induction assumption to ${ }^{i} T$ and conclude that $T$ is a $p$-group even for $i \neq 0$. If $i \neq 0$, Lemma 2.4 implies that $p\left({ }^{n-1} T\right)={ }^{i-1} T$. Then ${ }^{i-1} T$ is the direct sum of $2 x k_{1}$ cyclic subgroups of order $p^{j-1}$ and $2 x k_{2}$ cyclic subgroups of order $p^{j}$.

Now $o\left({ }^{i} T\right)=p^{2 x} \cdot o\left({ }^{i-1} T\right)$, and $o(T)=p^{2 x} \cdot o\left({ }^{n-1} T\right)$. Thus, letting $\sigma^{*}$ denote the restriction of $\sigma$ to ${ }^{n-1} T$, we see that $\operatorname{Ker}\left(\sigma^{*}\right)$ and $\operatorname{Ker}(\sigma)$ have the same order. Then $T$ and ${ }^{n-1} T$ both have the same number $k=2 x\left(k_{1}+k_{2}\right)$ of summands. By counting elements of order $p$, we see that, in general, no $p$-group may have fewer summands than any of its subgroups. ${ }^{i} T$ and ${ }^{i-1} T$ also have $k$ summands unless $j=1$. Assume $j=1$ so that ${ }^{i-1} T$ is the direct sum of $2 x k_{2}$ cyclic subgroups of order $p$. Applying the induction assumption to ${ }^{i} T$ and observing that $o\left({ }^{i} T\right)=p^{2 x} \cdot o\left({ }^{i-1} T\right)$, we see that either

(2.1) ${ }^{i} T$ is the direct sum of $2 x\left(k_{2}+1\right)$ cyclic subgroups of order $p$,

or

(2.2) ${ }^{i} T$ is the direct sum of $2 x\left(k_{2}-1\right)$ cyclic subgroups of order $p$ and of $2 x$ cyclic subgroups of order $p^{2}$.

Assume that (2.2) is satisfied, and apply the induction assumption to ${ }^{i+1} T,{ }^{i+2} T, \cdots,{ }^{n-2} T$. Since ${ }^{n-1} T$ has more summands than ${ }^{i} T$, there is an integer $l$ such that $0 \leqq l<n-1$ and ${ }^{i+l+1} T$ is isomorphic to the direct sum of ${ }^{i+l} T$ and of $2 x$ cyclic subgroups of order $p$. Then $\left({ }^{i+l+1} T\right) \sigma=\left({ }^{i+l} T\right) \sigma \neq 0$ which contradicts Lemma 2.4. We conclude that (2.1) is the only possibility for ${ }^{i} T$ when $j=1$.

If $j>1$, applying the induction assumption to ${ }^{i} T$, we see that

(2.3) ${ }^{i} T$ must be the direct sum of $2 x\left(k_{1}-1\right)$ cyclic subgroups of order $\mathrm{p}^{j-1}$ and of $2 x\left(k_{2}+1\right)$ cyclic subgroups of order $p^{j}$.

Since (2.1) is just a degenerate case of (2.3), we see that (2.3) must be satisfied whenever $T$ is not elemetary abelian. Then if $T$ is not elementary abelian, $T$ must contain a subgroup $S$ which is the direct sum of $2 x\left(k_{1}-1\right)$ cyclic subgroups of order $p^{j}$ and $2 x\left(k_{2}+1\right)$ cyclic subgroups of order $p^{j+1}$. Since $o(T)=o(S), T=S$, and the proof is complete.

We have also proved

LEMMA 2.7. For all $m \leqq n$, either ${ }^{m} T$ is elementary abelian, or else there exist nonnegative integers $j, k_{1}, k_{2}, x$ such that

(a) ${ }^{m-1} T$ is the direct sum of $2 x k_{1}$ cyclic subgroups of order $p^{j}$ and of $2 x k_{2}$ cyclic subgroups of order $p^{j+1}$; 
(b) ${ }^{m} T$ is the direct sum of $2 x\left(k_{1}-1\right)$ cyclic subgroups of order $p^{j}$ and of $2 x\left(k_{2}+1\right)$ cyclic subgroups of order $p^{j+1}$.

We now use Lemma 2.7 to obtain the following improvement of Theorem 2.6.

THEOREM 2.6A. Let $A=\boldsymbol{J}(T, \pi)$ be an n-uniform translation $H$-plane with invariant $r=p^{x}$ and translation group isomorphic to $T$. Then there exist integers $l, k$ with $0 \leqq l<k$ and subgroups $C_{i}$ of $T$ which satisfy the following conditions:

(a) $T=C_{1} \oplus \cdots \oplus C_{k}$;

(b) for $i \leqq l, C_{i}$ is the direct sum of $2 x$ cyclic subgroups of order $p^{j+1}$;

(c) for $i>l, C_{i}$ is the direct sum of $2 x$ cyclic subgroups of order $p^{j}$;

(d) for $i \leqq n=k j+l$,

$$
{ }^{i} 0=p^{q+1} \cdot\left(C_{1} \oplus \cdots \oplus C_{e}\right) \oplus p^{q} \cdot\left(C_{e+1} \oplus \cdots \oplus C_{k}\right)
$$

where $q$, e are given by $n-i=k q+e, 0 \leqq e<k$.

Proof. By Theorem 2.6, we have that $T$ is the direct sum of $2 x k_{1}$ cyclic subgroups of order $p^{j}$ and $2 x k_{2}$ cyclic subgroups of order $p^{j+1}$. Set $k=k_{1}+k_{2}$. Using Lemma 2.7 and Proposition 2.1 (8), it is easy to see that for $m \leqq k$,

$$
{ }^{m} T=D_{1} \oplus \cdots \oplus D_{m}
$$

where each $D_{i}$ is the direct sum of $2 x$ cyclic subgroups of order $p$. By Lemma 2.4, there exists an integer $c$ such that $p^{b} T={ }^{b-c} T$ for all $b \geqq c$. Clearly, $c=k$. Assume that for some $m$ with $0 \leqq m<$ $\min (k, n-k)$, there exist subgroups $E_{i}$ of $T$ satisfying

(2.4) ${ }^{m+k} T=E_{1} \oplus \cdots \oplus E_{m} \oplus D_{m+1} \oplus \cdots \oplus D_{k}$;

(2.5) $p E_{i}=D_{i}$ for $1 \leqq i \leqq m$;

(2.6) each $E_{i}$ is the direct sum of $2 x$ cyclic groups of order $p^{2}$.

Certainly the above requirements are satisfied for $m=0$. Let $\left\{d_{i}: 1 \leqq\right.$ $i \leqq 2 x\}$ be a basis for $D_{m+1}$. Since $\left.p^{(m+k+1} T\right)={ }^{m+1} T \supset D_{m+1}$, there exist $e_{i} \in{ }^{m+k+1} T$ satisfying $p e_{i}=d_{i}$. Let $E_{m+1}$ be the group generated by $\left\{e_{i}\right\}$. Suppose $e$ is an element of

$$
E_{m+1} \cap\left(E_{1} \oplus \cdots \oplus E_{m} \oplus D_{m+2} \oplus \cdots \oplus D_{k}\right) .
$$

Then by (2.5),

$$
p e \in D_{m+1} \cap\left(D_{1} \oplus \cdots \oplus D_{m}\right) .
$$

Then $p e=0$; hence 


$$
e \in D_{m+1} \cap\left(D_{1} \oplus \cdots \oplus D_{m} \oplus D_{m+2} \oplus \cdots \oplus D_{k}\right) .
$$

Then $e=0$. We have proved that the sum

$$
E=E_{1}+\cdots+E_{m+1}+D_{m+2}+\cdots+D_{k}
$$

is direct. We know $E \subset^{m+k+1} T$, and Proposition 2.1 (8) implies $E=$ ${ }^{m+k+1} T$. It is now easy to see that $(2.4)-(2.6)$ are all satisfied if we substitute $m+1$ for $m$.

Proceeding in this manner, we eventually obtain

$$
T=F_{1} \oplus \cdots \oplus F_{k}
$$

where $F_{i}$ is the direct sum of $2 x$ cyclic groups of order $p^{j+1}$ if $i \leqq k_{2}$ and of $2 x$ cyclic groups of order $p^{j}$ when $i>k_{2}$. The result now follows from setting $l=k_{2}, C_{i}=F_{l+1-i}$ for $1 \leqq i \leqq l$ and $C_{i}=F_{k+l+1-i}$ for $l<i \leqq k$. We may assure $l<k$ by changing the value of $j$ if necessary.

3. The translation groups of finite Desarguesian affine $H$-planes. The reader is referred to Klingenberg [5], [6] or Dembowski [2] for the definition of Desarguesian and Pappian affine $H$-planes as well as for all the results on such planes stated below. We do repeat the following definition.

DEFINITION 3.1. A Desarguesian $H$-ring (henceforth abbreviated to $H$-ring) is an associative ring with identity which satisfies the following three conditions:

(a) Every divisor of zero is a two-sided divisor of zero, and the set $N$ of divisors of zero is an ideal.

(b) Every nondivisor of zero has an inverse.

(c) If $n, m \in N$, then there is an $h \in H$ such that $n h=m$ or $n=m h$; and there is a $k \in H$ such that $k n=m$ or $n=k m$.

If $H$ denotes an $H$-ring, then Klingenberg defined [6] an incidence structure $\sum_{p}(H)$ as follows: points are left "homogeneous triples" of elements of $H$; lines are right "homogeneous triples"; a point and line are incident if and only if the inner products of their corresponding triples are zero. Klingenberg proved [6, S 28, S 29, proof of $S$ 29] that $\sum_{p}(H)$ is a projective $H$-plane whose affine $H$-planes are all isomorphic Desarguesian affine $H$-planes with translation groups isomorphic to $H^{+} \oplus H^{+}$. The affine $H$-planes belonging to $\Sigma_{p}(H)$ are themselves coordinatizable (in an affine manner) by the ring $H$ and Klingenberg denotes such an affine $H$-plane by $\Sigma_{a}(H)$. Call a projective $H$-plane $P$ Desarguesian if and only if $P$ is isomorphic to $\sum_{p}(H)$ for some $H$-ring $H$. 
By definition, all affine Desarguesian $H$-planes are translation $H$-planes. The author has proved [3, Theorem 5.4] that all finite $\sum_{p}(H)$ and hence also all finite $\sum_{a}(H)$ are $n$-uniform for various $n$. Let $\Sigma_{a}(H)$ or $\sum_{p}(H)$ be $n$-uniform with invariants $r, s, t$. Then $o(H)=s=r^{n}$ and $o(N)=t=r^{n-1}$ (See [3, Lemma 5.1].) It is clear from [3, Theorem 5.3 and Lemma 5.2 (1)] that $o\left(N^{i}\right)=r^{n-i}$ for $1 \leqq$ $i \leqq n$. In particular, $N$ is nilpotent of degree $n$. We are now in a position to state and prove the following algebraic corollary to Theorem 2.6.

CoRollary 3.1. Let $H$ be a finite $H$-ring with radical $N$. Let $r^{*}$ denote $o(H / N)$. Then $r^{*}$ is a prime power $p^{x}$. $H^{+}$is the direct sum of $x k_{1}$ cyclic subgroups of order $p^{j}$ and of $x k_{2}$ cyclic subgroups of order $p^{j+1}$ for some nonnegative integers $k_{1}, k_{2}, j$.

Proof. Since $H$ is a finite $H$-ring, $\sum_{a}(H)$ is an $n$-uniform translation $H$-plane. Since $r^{*}=o(H / N), r^{*}$ is the invariant of $\sum_{a}(H)$; hence $r^{*}$ is a prime power. The result now follows from Theorem 2.6 and the previous observation that the translation group of $\sum_{a}(H)$ is isomorphic to $H^{+} \oplus H^{+}$.

We remark that W. E. Clark and the author [1] have given an algebraic proof of Corollary 3.1. Nevertheless, it is interesting that the corollary should be an immediate consequence of a geometric theorem.

Lemma 3.2. Let $H$ be a finite $H$-ring with radical $N, \sum_{a}(H)$ be n-uniform. Then for each point $(c, d)$ of $\sum_{a}(H)$, one has ${ }^{i}(c, d)=$ $\left\{(c+a, d+b): a, b \in N^{n-i}\right\}, 0 \leqq i<n$.

Proof. Let $a \in N^{n-i}-N^{n-i+1}, b \in N^{n-j}-N^{n-j+1}$. We assume $i \geqq$ $j$. Let $[x, y]$ denote the line whose incident points are $\{(t x, t y): t \in H\}$. The lines through $(0,0)$ are the lines of the form $[x, y]$. (See [6, S23]. Note that $[x, y]$ is a line if and only if not both $x, y \in N$.) Let $[x, y]$ be a line through $(a, b)$. Then there exists $t_{0} \in H$ such that $a=t_{0} x, b=t_{0} y$; hence $x \in H-N$ and $t_{0} \in N^{n-i}$. Let $u \in H-N$, $w \in N^{i}, v=u x^{-1} y+w$. Then $[u, v]$ contains $(a, b)$. There are $(s-t) r^{n-i}$ satisfactory pairs $u, v$; and, since $[u, v]=\left[u^{\prime}, v^{\prime}\right]$ if and only if $u^{\prime}=$ $z u, v^{\prime}=z v$ for a unit $z$, these must give rise to at least $r^{n-i}$ distinct lines. Then $(a, b) \in \in^{i}(0,0)$. Similarly, if $j \geqq i,(a, b) \in \in^{j}(0,0)$. Let $X=$ $\left\{(a, b): a, b \in N^{n-i}\right\}$. Then $X \subset^{i}(0,0)$. Since $|X|=r^{2 i}=||^{i}(0,0) \mid,{ }^{i}(0,0)=$ $X$. This yields the result when $(c, d)=(0,0)$. To obtain the full result, one merely considers the translation $\tau(c, d)$ which maps each point $(x, y)$ to $(c+x, d+y)$. 
REMARK 3.3. Let $H, N, r^{*}=p^{x}, k_{1}, k_{2}$ be as in Corollary 3.1. Set $k=k_{1}+k_{2}$. Let $i$ be any nonnegative integer less than $n$ where $n$ satisfies $N^{n-1} \neq N^{n}=0$. Let $q, r$ be the nonnegative integers which satisfy $i=k q+r$ and $r<k$. Then $\left(N^{n-i}\right)^{+}$is the direct sum of $x(k-r)$ cyclic subgroups of order $p^{q}$ and of $x r$ cyclic subgroups of order $p^{q+1}$.

Proof. Let $T=H^{+} \oplus H^{+}$. Let $\pi$ be the set of lines $[x, y]$. It is clear from [6, S 23] that $\sum_{a}(H) \cong \boldsymbol{J}(T, \pi)$. By Lemma 3.2, $N^{n-i} \times$ $N^{n-i}={ }^{i}(0,0)={ }^{i} T$. Then the conclusion follows from Lemma 2.7 and Corollary 3.1.

In [1], W. E. Clark and the author prove the following result:

Proposition 3.4. Let there be given a prime integer $p$ and nonnegative integers $x, k_{1}, k_{2}, j$, such that $x>0$ and $k_{1} j+k_{2}(j+1)>0$. Then there exists a commutative $H$-ring $H$ with radical $N$ such that $o(H / N)=p^{x}$ and so that $H^{+}$is tha direct sum of $x k_{1}$ cyclic subgroups of order $p^{j}$ and of $x k_{2}$ cyclic subgroups of order $p^{j+1}$.

Klingenberg proves (See [5] or [2].) that if $H$ is a commutative $H$-ring, then $\sum_{a}(H)$ is Pappian. We then obtain the following strong converse to Theorem 2.6 as an immediate corollary to Proposition 3.4.

CoROllary 3.5. Let $r=p^{x}, k_{1}, k_{2}, j$ be given with $k_{1} j+k_{2}(j+1)>$ $0, x>0$. Then there exists a Pappian affine $H$-plane with invariant $r$ whose translation group is the direct product of $2 x k_{1}$ cyclic subgroups of order $p^{j}$ and $2 x k_{2}$ cyclic subgroups of order $p^{j+1}$.

Corollary 3.5 says that all translation groups of $n$-uniform translation $H$-planes can be obtained as translation groups of Pappian $H$-planes. Actually it says somewhat more: namely, if $T$ is the translation group of an $n$-uniform translation $H$-plane $A$ whose invariant is $p^{x}$, then $T$ can be obtained as the translation group of a Pappian affine $H$-plane $B$ whose invariant is $p^{y}$ where $y$ is any positive integer such that $y \mid x k_{1}$ and $y \mid x k_{2}$. In particular, one can always take $y=x$ so that $A$ and $B$ will have the same invariant. Also all translation groups can be obtained as the translation groups of Pappian affine $H$-planes whose associated affine planes are of prime order.

\section{REFERENCES}

1. W. E. Clark and D. A. Drake, Finite chain rings, (To appear) 
2. P. Dembowski, Finite Geometries, Ergebnisse der Mathematik 44, Springer, BerlinHeidelberg-New York, 1968.

3. D. A. Drake, On n-uniform Hjelmslev planes, J. Combinatorial Theory 9 (1970), 267-288.

4. E. Kleinfeld, Finite Hjelmslev planes, Illinois J. Math. 3 (1959), 403-407.

5. W. Klingenberg, Projektive und affine Ebenen mit Nachbarelementen, Math. Z.. 60 (1954), 384-406.

6. - Desarguessche Ebenen mit Nachbarelementen, Abh. Math. Sem. Univ. Hamburg, 20 (1955), 97-111.

7. H. Lüneburg, Affine Hjelmslev-Ebenen mit transitiver Translationsgruppe, Math.

Z. 79 (1962), 260-288.

Received August 19, 1970.

UNIVERSITY OF FLORIDA 



\section{PACIFIC JOURNAL OF MATHEMATICS}

\section{EDITORS}

\author{
H. SAMELSON \\ Stanford University \\ Stanford, California 94305 \\ C. R. HовBY \\ University of Washington \\ Seattle, Washington 98105
}

J. DUGUndis

Department of Mathematics

University of Southern California

Los Angeles, California 90007

RICHARD ARENS

University of California

Los Angeles, California 90024

\section{ASSOCIATE EDITORS}
E. F. BeCKenbaCH
B. H. NeUmanN
F. WOLF
K. YoshidA

\section{SUPPORTING INSTITUTIONS}

\author{
UNIVERSITY OF BRITISH COLUMBIA \\ CALIFORNIA INSTITUTE OF TECHNOLOGY \\ UNIVERSITY OF CALIFORNIA \\ MONTANA STATE UNIVERSITY \\ UNIVERSITY OF NEVADA \\ NEW MEXICO STATE UNIVERSITY \\ OREGON STATE UNIVERSITY \\ UNIVERSITY OF OREGON \\ OSAKA UNIVERSITY \\ UNIVERSITY OF SOUTHERN CALIFORNIA
}

\author{
STANFORD UNIVERSITY \\ UNIVERSITY OF TOKYO \\ UNIVERSITY OF UTAH \\ WASHINGTON STATE UNIVERSITY \\ UNIVERSITY OF WASHINGTON

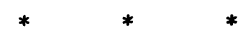 \\ AMERICAN MATHEMATICAL SOCIETY \\ CHEVRON RESEARCH CORPORATION \\ NAVAL WEAPONS CENTER
}

The Supporting Institutions listed above contribute to the cost of publication of this Journal, but they are not owners or publishers and have no responsibility for its content or policies.

Mathematical papers intended for publication in the Pacific Journal of Mathematics should be in typed form or offset-reproduced, (not dittoed), double spaced with large margins. Underline Greek letters in red, German in green, and script in blue. The first paragraph or two must be capable of being used separately as a synopsis of the entire paper. The editorial "we" must not be used in the synopsis, and items of the bibliography should not be cited there unless absolutely necessary, in which case they must be identified by author and Journal, rather than by item number. Manuscripts, in duplicate if possible, may be sent to any one of the four editors. Please classify according to the scheme of Math. Rev. Index to Vol. 39. All other communications to the editors should be addressed to the managing editor, Richard Arens, University of California, Los Angeles, California, 90024.

50 reprints are provided free for each article; additional copies may be obtained at cost in multiples of 50 .

The Pacific Journal of Mathematics is published monthly. Effective with Volume 16 the price per volume (3 numbers) is $\$ 8.00$; single issues, $\$ 3.00$. Special price for current issues to individual faculty members of supporting institutions and to individual members of the American Mathematical Society: $\$ 4.00$ per volume; single issues $\$ 1.50$. Back numbers are available.

Subscriptions, orders for back numbers, and changes of address should be sent to Pacific Journal of Mathematics, 103 Highland Boulevard, Berkeley, California, 94708.

PUBLISHED BY PACIFIC JOURNAL OF MATHEMATICS, A NON-PROFIT CORPORATION

Printed at Kokusai Bunken Insatsusha (International Academic Printing Co., Ltd.), 7-17, Fujimi 2-chome, Chiyoda-ku, Tokyo, Japan. 


\section{Pacific Journal of Mathematics}

Vol. 38, No. $2 \quad$ April, 1971

Richard Davis Anderson and Thomas Ashland Chapman, Extending

homeomorphisms to Hilbert cube manifolds .................. 281

Nguyen Huu Anh, Restriction of the principal series of $\operatorname{SL}(n, \mathbf{C})$ to some

reductive subgroups................................ 295

David W. Boyd, Indices for the Orlicz spaces . . . . . . . . . . . . 315

William Garfield Bridges, The polynomial of a non-regular digraph ...... 325

Billie Chandler Carlson, Robert K. Meany and Stuart Alan Nelson, Mixed

arithmetic and geometric means........................ 343

H. A. Çelik, Commutative associative rings and anti-flexible rings ...... 351

Hsin Chu, On the structure of almost periodic transformation groups ...... 359

David Allyn Drake, The translation groups of n-uniform translation

Hjelmslev planes ................................ 365

Michael Benton Freeman, The polynomial hull of a thin two-manifold . . . . 377

Anthony Alfred Gioia and Donald Goldsmith, Convolutions of arithmetic

functions over cohesive basic sequences .................... 391

Leslie C. Glaser, A proof of the most general polyhedral Schoenflies

conjecture possible ................................

Thomas Lee Hayden and Ted Joe Suffridge, Biholomorphic maps in Hilbert

space have a fixed point ................................ 419

Roger Alan Horn, Schlicht mappings and infinitely divisible kernels ...... 423

Norman Ray Howes, On completeness ...................... 431

Hideo Imai, Sario potentials on Riemannian spaces................ 441

A. A. Iskander, Subalgebra systems of powers of partial universal

algebras.

Barry E. Johnson, Norms of derivations of $\mathscr{L}(\mathrm{X})$.

David Clifford Kay and Eugene W. Womble, Axiomatic convexity theory and relationships between the Carathéodory, Helly, and Radon numbers

Constantine G. Lascarides, A study of certain sequence spaces of Maddox

and a generalization of a theorem of Iyer .............

C. N. Linden, On Blaschke products of restricted growth .

John S. Lowndes, Some triple integral equations ................. 515

Declan McCartan, Bicontinuous preordered topological spaces ......... 523

S. Moedomo and J. Jerry Uhl, Jr., Radon-Nikodým theorems for the Bochner and Pettis integrals ...

Calvin Cooper Moore and Joseph Albert Wolf, Totally real representations

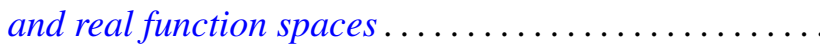

Reese Trego Prosser, A form of the moment problem for Lie groups. ... 